\title{
Poincaré series and monodromy of a two-dimensional quasihomogeneous hypersurface singularity
}

\author{
Wolfgang Ebeling \\ Institut für Mathematik, Universität Hannover, \\ Postfach 6009, D-30060 Hannover, Germany \\ E-mail: ebeling@math.uni-hannover.de
}

\begin{abstract}
A relation is proved between the Poincaré series of the coordinate algebra of a two-dimensional quasihomogeneous isolated hypersurface singularity and the characteristic polynomial of its monodromy operator. For a Kleinian singularity not of type $A_{2 n}$, this amounts to the statement that the Poincare series is the quotient of the characteristic polynomial of the Coxeter element by the characteristic polynomial of the affine Coxeter element of the corresponding root system. We show that this result also follows from the McKay correspondence.
\end{abstract}

\section{Introduction}

S. M. Gusein-Zade, F. Delgado, and A. Campillo GDC have shown that for an irreducible plane curve singularity the Poincare series of the ring of functions on the curve coincides with the zeta function of its monodromy transformation.

In this paper we show that there is also a relation between the Poincaré series of the coordinate algebra of a two-dimensional quasihomogeneous isolated hypersurface singularity and the characteristic polynomial of its monodromy operator.

Let $(X, x)$ be a normal surface singularity with good $\mathbb{C}^{*}$-action. The coordinate algebra $A$ is a graded algebra. We consider the Poincaré series $p_{A}(t)$ of $A$. Let $\left\{g ; b ;\left(\alpha_{1}, \beta_{1}\right), \ldots,\left(\alpha_{r}, \beta_{r}\right)\right\}$ be the orbit invariants of $(X, x)$. We define

$$
\begin{aligned}
& \psi_{A}(t):=(1-t)^{2-r} \prod_{i=1}^{r}\left(1-t^{\alpha_{i}}\right), \\
& \phi_{A}(t):=p_{A}(t) \psi_{A}(t) .
\end{aligned}
$$

Let $(X, x)$ be a hypersurface singularity. Then $\phi_{A}(t)$ is a product of cyclotomic polynomials.

K. Saito Sa3, Sa4 has introduced a duality between polynomials which are products of cyclotomic polynomials. He has shown that V. I. Arnold's strange duality between the 14 exceptional unimodal hypersurface singularities is related 
to such a duality between the characteristic polynomials of the monodromy operators of the singularities. It is now well-known that Arnold's strange duality is related to the mirror symmetry of $K 3$ surfaces (see e.g. (D5)).

The main results of the paper are the following. We show that the dual (in Saito's sense) of the rational function $\tilde{\phi}_{A}(t):=\phi_{A}(t) /(1-t)^{2 g}$ is the characteristic polynomial of the monodromy operator of $(X, x)$ (Theorem 11). Similar results can be proved for isolated complete intersection singularities (abbreviated ICIS in the sequel) of certain types (see Theorem 3 and 4 ).

If $(X, x)$ is a Kleinian singularity not of type $A_{2 n}$, then $\psi_{A}(t)$ is the characteristic polynomial of the affine Coxeter element of the corresponding root system and the above result implies that $\phi_{A}(t)$ is the characteristic polynomial of the Coxeter element. Hence the Poincaré series of a polyhedral group which is not a cyclic group of odd order is the quotient of these two polynomials. We derive this result also directly from the McKay correspondence using ideas of the paper [Kos]. There are various formulas for Poincaré series of binary polyhedral groups in $\mathrm{Kn}$, Kos, $\mathrm{Sp}$, but this relation seems to be new.

The author thanks K. Hulek for useful discussions. He is grateful to E. Brieskorn for pointing out an error in an earlier version of the paper.

\section{Main results}

Let $(X, x)$ be a normal surface singularity with a good $\mathbb{C}^{*}$-action. So $X$ is a normal two-dimensional affine algebraic variety over $\mathbb{C}$ which is smooth outside its vertex $x$. Its coordinate ring $A$ has the structure of a graded $\mathbb{C}$-algebra $A=\bigoplus_{k=0}^{\infty} A_{k}, A_{0}=\mathbb{C}$, and $x$ is defined by the maximal ideal $\mathfrak{m}=\bigoplus_{k=1}^{\infty} A_{k}$.

According to I. Dolgachev [D2], there exist a simply connected Riemann surface $\mathcal{D}$, a discrete cocompact subgroup $\Gamma$ of $\operatorname{Aut}(\mathcal{D})$ and a line bundle $\mathcal{L}$ on $\mathcal{D}$ to which the action of $\Gamma$ lifts such that

$$
A_{k}=H^{0}\left(\mathcal{D}, \mathcal{L}^{k}\right)^{\Gamma} .
$$

Let $Z:=\mathcal{D} / \Gamma$. By $[\mathrm{P}$, Theorem 5.1] (see also [W2, Theorem 5.4.1]), there exist a divisor $D_{0}$ on $Z, p_{1}, \ldots, p_{r} \in Z$, and integers $\alpha_{i}, \beta_{i}$ with $0<\beta_{i}<\alpha_{i}$ and $\left(\alpha_{i}, \beta_{i}\right)=1$ for $i=1, \ldots, r$ such that

$$
A_{k}=L\left(k D_{0}+\sum_{i=1}^{r}\left[k \frac{\alpha_{i}-\beta_{i}}{\alpha_{i}}\right] p_{i}\right) .
$$

Here $[x]$ denotes the largest integer $\leq x$, and $L(D)$ for a divisor $D$ on $Z$ denotes the linear space of meromorphic functions $f$ on $Z$ such that $(f) \geq-D$. We number the points $p_{i}$ so that $\alpha_{1} \leq \alpha_{2} \leq \ldots \leq \alpha_{r}$. Let $g$ be the genus of $Z$ and define $b:=$ degree $D_{0}+r$. Then $\left\{g ; b ;\left(\alpha_{1}, \beta_{1}\right), \ldots,\left(\alpha_{r}, \beta_{r}\right)\right\}$ are called the orbit invariants of $(X, x)$, cf. e.g. W3]. Define $\operatorname{vdeg}(\mathcal{L}):=-b+\sum_{i=1}^{r} \frac{\beta_{i}}{\alpha_{i}}$.

Now assume that $(X, x)$ is Gorenstein. By D4, there exists an integer $R$ such that $\mathcal{L}^{-R}$ and the tangent bundle $T_{\mathcal{D}}$ of $\mathcal{D}$ are isomorphic as $\Gamma$-bundles and

$$
\begin{aligned}
R \cdot \operatorname{vdeg}(\mathcal{L}) & =2-2 g-r+\sum_{i=1}^{r} \frac{1}{\alpha_{i}} \\
R \beta_{i} & \equiv 1 \bmod \alpha_{i}, \quad i=1, \ldots, r .
\end{aligned}
$$


Following [D3, 3.3.15] we call $R$ the exponent of $(X, x)$. Since $b$ and the $\beta_{i}$ are determined by the $\alpha_{i}$ and the number $R$, we write the orbit invariants also as $g ; \alpha_{1}, \ldots, \alpha_{r}$.

The Gorenstein surface singularities with good $\mathbb{C}^{*}$-action fall into three classes $\mathrm{D} 4, \mathrm{~W} 3$.

(1) $\mathcal{D}=\mathbb{P}^{1}(\mathbb{C})$ : Then $R=-2$ or $R=-1, g=0$, and $(X, x)$ is a Kleinian singularity.

(2) $\mathcal{D}=\mathbb{C}$ : Then $R=0, r=0$, and $g=1$. Hence $(X, x)$ is a simply elliptic singularity Sa1].

(3) $\mathcal{D}=\mathbb{H}$, the upper half plane: The remaining Gorenstein surface singularities with good $\mathbb{C}^{*}$-action belong to this class. We have $R \geq 1$.

We consider the Poincaré series of the algebra $A$

$$
p_{A}(t)=\sum_{k=0}^{\infty} a_{k} t^{k}
$$

where $a_{k}=\operatorname{dim} A_{k}$. It is well known that $p_{A}(t)$ is a rational function and the the order of the pole of $p_{A}(t)$ at $t=1$ is equal to the dimension of $A$, hence equal to 2 . Moreover, $p_{A}(t)$ has simple poles at the $\alpha_{i}$-th roots of unity different from 1.

We define

$$
\begin{aligned}
\psi_{A}(t) & :=(1-t)^{2-r} \prod_{i=1}^{r}\left(1-t^{\alpha_{i}}\right), \\
\phi_{A}(t) & :=p_{A}(t) \psi_{A}(t) \\
\tilde{\phi}_{A}(t) & :=\frac{\phi_{A}(t)}{(1-t)^{2 g}} .
\end{aligned}
$$

Then $\phi_{A}(t)$ is a polynomial.

Now let $(X, x)$ be an ICIS with weights $q_{1}, \ldots, q_{n}$ and degrees $d_{1}, \ldots, d_{n-2}$. Then its Poincaré series is given by (see e.g. [W3, Proposition (2.2.2)])

$$
p_{A}(t)=\frac{\prod_{i=1}^{n-2}\left(1-t^{d_{i}}\right)}{\prod_{j=1}^{n}\left(1-t^{q_{j}}\right)} .
$$

Hence $p_{A}(t), \psi_{A}(t), \phi_{A}(t)$, and $\tilde{\phi}_{A}(t)$ are rational functions of the form

$$
\phi(t)=\prod_{m \mid h}\left(1-t^{m}\right)^{\chi_{m}} \quad \text { for } \chi_{m} \in \mathbb{Z} \text { and for some } h \in \mathbb{N} .
$$

Given a rational function

$$
\phi(t)=\prod_{m \mid h}\left(1-t^{m}\right)^{\chi_{m}},
$$

K. Saito Sa3 has defined a dual rational function

$$
\phi^{*}(t)=\prod_{k \mid h}\left(1-t^{k}\right)^{-\chi_{h / k}} .
$$

We are now able to state the main results of the paper. 
Theorem 1 Let $(X, x)$ be a quasihomogeneous hypersurface singularity in $\mathbb{C}^{3}$. Then $\tilde{\phi}_{A}^{*}(t)$ is the characteristic polynomial of the classical monodromy operator of $(X, x)$.

The proof of Theorem 11 will be given in Section 2 .

Let $(X, x)$ be a Kleinian singularity. Then $g=0$, and therefore $\tilde{\phi}_{A}(t)=$ $\phi_{A}(t)$. It is well known that the Kleinian singularities correspond to root systems of type $A_{l}, D_{l}, E_{6}, E_{7}$, or $E_{8}$. The classical monodromy operator of $(X, x)$ is the Coxeter element of the corresponding root system. The polynomial $\psi_{A}(t)$ is the characteristic polynomial of the affine Coxeter element of the corresponding root system (see [St, p. 591] or [Sp, 6.2], if $(X, x)$ is not of type $\left.A_{l}\right)$. In the case $A_{2 n}$, we have

$$
\phi_{A}(t)=\frac{1-t^{4 n+2}}{1-t^{2}} .
$$

In this case, $\phi_{A}^{*}(t) \neq \phi_{A}(t)$. In all other cases one can verify that $\phi_{A}^{*}(t)=\phi_{A}(t)$ (cf. Table 1). Therefore we obtain from Theorem 11:

Theorem 2 Let $(X, x)$ be a Kleinian singularity not of type $A_{2 n}$. Then $\phi_{A}(t)$ and $\psi_{A}(t)$ are the characteristic polynomials of the Coxeter element and the affine Coxeter element respectively of the corresponding root system. Hence $p_{A}(t)$ is the quotient of these polynomials.

In Section 3 we shall give a direct proof of Theorem 2 using the McKay correspondence.

\section{Poincaré series and monodromy}

In this section we shall prove Theorem 1 .

Let $(X, x)$ be a Gorenstein surface singularity with a good $\mathbb{C}^{*}$-action. The residue of the Poincaré series $p_{A}(t)$ at a primitive $\alpha_{i}$-th root of unity can be computed as follows.

Proposition 1 Let $(X, x)$ be Gorenstein and $R$ be the exponent of $(X, x)$. Let $\xi_{i}=\exp \left(2 \pi \sqrt{-1} / \alpha_{i}\right)$. Then the residue of $p_{A}(t)$ at $t=\xi_{i}$ is equal to

$$
\sum_{\alpha_{i} \mid \alpha_{j}} \frac{\xi_{i} \cdot \xi_{i}^{R}}{\alpha_{j}\left(1-\xi_{i}^{R}\right)} .
$$

From Proposition 1 we can derive the following proposition generalizing $\mathrm{W} 1$, Proposition (2.8)]). For integers $a_{1}, \ldots, a_{r}$ we denote by $\left\langle a_{1}, \ldots, a_{r}\right\rangle$ their least common multiple and by $\left(a_{1}, \ldots, a_{r}\right)$ their greatest common divisor.

Proposition 2 Let $A$ be the coordinate algebra of a Gorenstein surface singularity with good $\mathbb{C}^{*}$-action. Suppose that $A$ is generated by 3 elements of weights $q_{1}, q_{2}, q_{3}$. For each $i=1, \ldots, r$ let $\xi_{i}=\exp \left(2 \pi \sqrt{-1} / \alpha_{i}\right)$. Then the Poincaré series is given by

$$
p_{A}(t)=\frac{\left(1-t^{d}\right)}{\left(1-t^{q_{1}}\right)\left(1-t^{q_{2}}\right)\left(1-t^{q_{3}}\right)}
$$

if and only if the following conditions hold: 
(a) $\left(q_{1}, q_{2}, q_{3}\right)=1$.

(b) $2 g-2+r-\sum_{i=1}^{r} \frac{1}{\alpha_{i}}=\frac{R d}{q_{1} q_{2} q_{3}}$.

(c) For each $i$

$$
\sum_{\alpha_{i} \mid \alpha_{j}} \frac{\xi_{i} \cdot \xi_{i}^{R}}{\alpha_{j}\left(1-\xi_{i}^{R}\right)}= \begin{cases}\frac{-\xi_{i}\left(1-\xi_{i}^{d}\right)}{q_{i_{1}}\left(1-\xi_{i}^{q_{2}}\right)\left(1-\xi_{i}^{q_{3}}\right)} & \text { if } \alpha_{i} \mid q_{i_{1}} \text { and } \alpha_{i} \backslash q_{i_{2}}, q_{i_{3}}, \\ \frac{-d \xi_{i}}{q_{i_{1}} q_{i_{2}}\left(1-\xi_{i}^{q_{3}}\right)} & \text { if } \alpha_{i}\left|q_{i_{1}}, \alpha_{i}\right| q_{i_{2}}, \alpha_{i} \backslash q_{i_{3}} .\end{cases}
$$

(d) For all $i$ and $j$ so that $i \neq j,\left(q_{i}, q_{j}\right) \mid d$.

(e) $d=q_{1}+q_{2}+q_{3}+R$.

Let $(X, x)$ be an isolated hypersurface singularity in $\mathbb{C}^{3}$ given by a quasihomogeneous equation $f\left(z_{1}, z_{2}, z_{3}\right)=0$ of degree $d$ and weights $q_{1}, q_{2}, q_{3}$. Then the Poincaré series is given by

$$
p_{A}(t)=\frac{\left(1-t^{d}\right)}{\left(1-t^{q_{1}}\right)\left(1-t^{q_{2}}\right)\left(1-t^{q_{3}}\right)} .
$$

From Proposition 2 one can easily derive the following proposition which was proven by P. Orlik and P. Wagreich [OW, 3.6 Proposition 1] using another method.

Proposition 3 (Orlik,Wagreich) Let $(X, x)$ be a quasihomogeneous isolated hypersurface singularity. Let $w_{i}=d / q_{i}=u_{i} / v_{i}$ where $\left(u_{i}, v_{i}\right)=1$ und $u_{i}, v_{i} \geq$ 1. Assume $1 \leq v_{1} \leq v_{2} \leq v_{3}$. Then the table below indicates the number of orbit invariants $\alpha$ of each type:

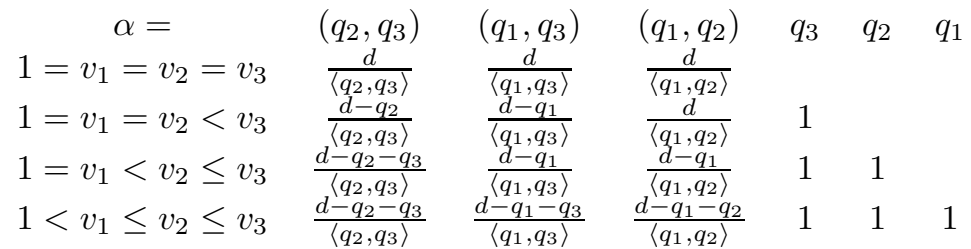

The blank entries are zero if $q_{i}$ does not divide $q_{j}$ for $j \neq i$. If $q_{i} \mid q_{j}$, then $\left(q_{i}, q_{j}\right)=q_{i}$ and we list those orbit invariants under the column headed $\left(q_{i}, q_{j}\right)$.

On the other hand, we consider the characteristic polynomial of the monodromy operator of $(X, x)$. Let $\mathcal{X}_{t}$ be a Milnor fibre of the singularity $(X, x)$ and denote by $M:=H_{2}\left(\mathcal{X}_{t}, \mathbb{Z}\right)$ the corresponding Milnor lattice. Let $c: M \rightarrow M$ be the classical monodromy operator of the singularity $(X, x)$. It is well-known that $c$ is quasi-unipotent and therefore the eigenvalues of $c$ are roots of unity. We write the characteristic polynomial $\phi_{M}(t)=\operatorname{det}(t I-c)$ of $c$ as

$$
\phi_{M}(t)=\prod_{m \mid h}\left(t^{m}-1\right)^{\chi_{m}} \quad \text { for } \chi_{m} \in \mathbb{Z} \text { and for some } h \in \mathbb{N} \text {. }
$$

The characteristic polynomial $\phi_{M}(t)$ can be computed as follows [MO] (see also $\mathrm{Sa} 4)$. Consider the rational function

$$
\Phi(T):=T^{-d} \frac{\left(T^{d}-T^{q_{1}}\right)\left(T^{d}-T^{q_{2}}\right)\left(T^{d}-T^{q_{3}}\right)}{\left(T^{q_{1}}-1\right)\left(T^{q_{2}}-1\right)\left(T^{q_{3}}-1\right)} .
$$


By [Sa2, (1.3) Theorem] there exist finitely many integers $m_{1}, \ldots, m_{\mu}$ such that

$$
\Phi(T)=T^{m_{1}}+\ldots+T^{m_{\mu}} .
$$

Then $\omega_{i}:=\exp \left(2 \pi \sqrt{-1} m_{i} / d\right), i=1, \ldots, \mu$, are the zeros of $\phi_{M}(t)$. Put $\Lambda_{k}:=$ $\omega_{1}^{k}+\ldots+\omega_{\mu}^{k}$ for $k \in \mathbb{N}$ and let $\omega:=\exp (2 \pi \sqrt{-1} / d)$. Then one has $\Lambda_{k}=\Phi\left(\omega^{k}\right)$. From this one can derive that

$$
\Lambda_{k}=\left(\delta\left(k q_{1} \bmod d\right) \frac{d}{q_{1}}-1\right)\left(\delta\left(k q_{2} \bmod d\right) \frac{d}{q_{2}}-1\right)\left(\delta\left(k q_{3} \bmod d\right) \frac{d}{q_{3}}-1\right)
$$

where $\delta$ is the delta function, i.e., $\delta(0):=1$ and $\delta(x):=0$ for $x \neq 0$. The numbers $\Lambda_{k}$ and $\chi_{m}$ are related by the formula

$$
\Lambda_{k}=\sum_{m \mid k} m \chi_{m}
$$

Proof of Theorem 团. We have

$$
\tilde{\phi}_{A}(t)=\frac{\left(1-t^{d}\right)\left(1-t^{\alpha_{1}}\right) \cdots\left(1-t^{\alpha_{r}}\right)}{(1-t)^{2 g-2+r}\left(1-t^{q_{1}}\right)\left(1-t^{q_{2}}\right)\left(1-t^{q_{3}}\right)} .
$$

From Proposition 3 we conclude that $q_{i} \mid d$ or $q_{i}=\alpha_{j}$ for some $j, 1 \leq j \leq r$, and that $\alpha_{i} \mid d$ or $\alpha_{i}=q_{j}$ for some $j, 1 \leq j \leq 3$. Therefore we may assume that

$$
\tilde{\phi}_{A}(t)=\frac{\left(1-t^{d}\right) \prod_{\alpha_{i} \mid d}\left(1-t^{\alpha_{i}}\right)}{(1-t)^{2 g-2+r} \prod_{q_{j} \mid d}\left(1-t^{q_{j}}\right)} .
$$

Hence

$$
\tilde{\phi}_{A}^{*}(t)=\frac{\left(1-t^{d}\right)^{2 g-2+r} \prod_{q_{j} \mid d}\left(1-t^{d / q_{j}}\right)}{(1-t) \prod_{\alpha_{i} \mid d}\left(1-t^{d / \alpha_{i}}\right)} .
$$

Denote by $\tilde{\Lambda}_{k}$ the sum of the $k$-th powers of the roots of $\tilde{\phi}_{A}^{*}(t)=0$. For the proof of Theorem 1 we must show that $\tilde{\Lambda}_{k}=\Lambda_{k}$ for all $k \in \mathbb{N}$.

(a) We first have

$$
\tilde{\Lambda}_{1}=-1=\Lambda_{1} \text {. }
$$

(b) Now suppose that $k q_{i_{1}} \equiv 0 \bmod d$ but $k q_{i_{2}} \not \equiv 0 \bmod d, k q_{i_{3}} \not \equiv 0 \bmod d$. Then we claim that for all $i$ with $\alpha_{i} \mid d$ the number $\frac{d}{\alpha_{i}}$ does not divide $k$. For suppose the contrary. By Proposition 3 we have $\alpha_{i}=\left(q_{j_{1}}, q_{j_{2}}\right)$ or $\alpha_{i}=q_{j_{1}}$. Now $\frac{d}{\left(q_{j_{1}}, q_{j_{2}}\right)} \mid k$ implies $d \mid k q_{j_{1}}$ and $d \mid k q_{j_{2}}$, which contradicts our assumption. But $\alpha_{i}=q_{j_{1}}$ is only possible if $\alpha_{i} \not d$. Therefore we have shown

$$
\tilde{\Lambda}_{k}=-1+\frac{d}{q_{i_{1}}}=\Lambda_{k} .
$$

(c) We now consider the case that $k q_{i_{1}} \equiv 0 \bmod d, k q_{i_{2}} \equiv 0 \bmod d, k q_{i_{3}} \not \equiv$ $0 \bmod d$. By Proposition 3 and the same arguments as in (b), we see that $\alpha_{i} \mid d$ and $\frac{d}{\alpha_{i}} \mid k$ only if $\alpha_{i}=\left(q_{i_{1}}, q_{i_{2}}\right)$. By Proposition 3 we therefore get

$$
\tilde{\Lambda}_{k}=-1+\delta\left(d \bmod q_{i_{1}}\right) \frac{d}{q_{i_{1}}}+\delta\left(d \bmod q_{i_{2}}\right) \frac{d}{q_{i_{2}}}-\sum_{\alpha_{j}=\left(q_{i_{1}}, q_{i_{2}}\right)} \frac{d}{\alpha_{j}}
$$




$$
\begin{aligned}
& =-1+\frac{d}{q_{i_{1}}}+\frac{d}{q_{i_{2}}}-\frac{d^{2}}{q_{i_{1}} q_{i_{2}}} \\
& =-\left(\frac{d}{q_{i_{1}}}-1\right)\left(\frac{d}{q_{i_{2}}}-1\right) \\
& =\Lambda_{k} .
\end{aligned}
$$

(d) Finally, assume that $k q_{1} \equiv 0 \bmod d, k q_{2} \equiv 0 \bmod d$, and $k q_{3} \equiv 0 \bmod d$. Since the greatest common divisor of $q_{1}, q_{2}, q_{3}$ is 1 , it follows that $d \mid k$. Then we have

$$
\begin{aligned}
\tilde{\Lambda}_{k} & =(2 g-2+r) d+\sum_{j=1}^{3} \delta\left(d \bmod q_{j}\right) \frac{d}{q_{j}}-\sum_{i=1}^{r} \delta\left(d \bmod \alpha_{i}\right) \frac{d}{\alpha_{i}}-1 \\
& =(2 g-2+r) d+\sum_{j=1}^{3} \frac{d}{q_{j}}-\sum_{i=1}^{r} \frac{d}{\alpha_{i}}-1 .
\end{aligned}
$$

By Proposition 2(b) and (e) we get

$$
\begin{aligned}
\tilde{\Lambda}_{k} & =\frac{R d^{2}}{q_{1} q_{2} q_{3}}+\sum_{j=1}^{3} \frac{d}{q_{j}}-1 \\
& =\frac{d^{2}\left(d-q_{1}-q_{2}-q_{3}\right)}{q_{1} q_{2} q_{3}}+\sum_{j=1}^{3} \frac{d}{q_{j}}-1 \\
& =\left(\frac{d}{q_{1}}-1\right)\left(\frac{d}{q_{2}}-1\right)\left(\frac{d}{q_{3}}-1\right) \\
& =\Lambda_{k} .
\end{aligned}
$$

This completes the proof of Theorem 1 .

\section{The McKay correspondence}

In this section we shall derive Theorem 2 from the McKay correspondence.

Let $(X, x)$ be a Kleinian singularity. Then $\mathcal{D}=\mathbb{P}^{1}(\mathbb{C})$, and $\Gamma$ is a finite subgroup of $\operatorname{Aut}\left(\mathbb{P}^{1}(\mathbb{C})\right)=P G L(2, \mathbb{C})$. We may assume that $\Gamma \subset P S U(2) \cong$ $S O(3)$. Up to conjugacy, there are five classes of such groups: (1) $\mathcal{C}_{l+1}$, the cyclic group of order $l+1, l \geq 1,(2) \mathcal{D}_{l-2}$, the dihedral group of order $2(l-2)$, $l \geq 4$, (3) $\mathcal{T}$, the tetrahedral group of order 12 , (4) $\mathcal{O}$, the octahedral group of order $24,(5) \mathcal{I}$, the icosahedral group of order 60 . It is well known that there is a correspondence between these singularities and the irreducible root systems as indicated in Table 1 .

Let $\nu=-\frac{2}{R}$. We have $A_{k}=S^{\nu k}\left(\mathbb{C}^{2}\right)^{\Gamma}$ where $S^{\nu k}\left(\mathbb{C}^{2}\right)$ denotes the $\nu k$ th symmetric power of $\mathbb{C}^{2}$ and the action of $\Gamma$ on $S^{\nu k}\left(\mathbb{C}^{2}\right)$ is induced by its action on $\mathbb{P}^{1}(\mathbb{C})$. We relate the series $p_{A}(t)$ to another Poincaré series which is considered in $\operatorname{Kos}$. Let $S U(2) \rightarrow P S U(2)$ be the usual double covering and let $G \subset S U(2)$ be the inverse image of $\Gamma \subset P S U(2)$. Let $\rho_{m}$ be the representation of $G$ on $S^{m}\left(\mathbb{C}^{2}\right)$ induced by its action on $\mathbb{C}^{2}$. Let $\gamma_{0}, \ldots, \gamma_{l}$ be the equivalence classes of irreducible finite dimensional complex representations of $G$ where $\gamma_{0}$ is the class of the trivial representation. For each integer $m \geq 0$ we have a 
Table 1: Kleinian singularities

\begin{tabular}{|c|c|c|c|c|}
\hline & $\Gamma$ & $g ; \alpha_{1}, \ldots, \alpha_{r}$ & Weights & $\pi_{A}$ \\
\hline$A_{2 n-1}$ & $\mathcal{C}_{2 n}$ & $0 ; n, n$ & $1, n, n / 2 n$ & $2 n / 1$ \\
$A_{2 n}$ & $\mathcal{C}_{2 n+1}$ & $0 ; 2 n+1,2 n+1$ & $2,2 n+1,2 n+1 / 4 n+2$ & $4 n+2 / 2$ \\
$D_{l}$ & $\mathcal{D}_{l-2}$ & $0 ; 2,2, l-2$ & $2, l-2, l-1 / 2(l-1)$ & $2 \cdot 2(l-1) / 1 \cdot(l-1)$ \\
$E_{6}$ & $\mathcal{T}$ & $0 ; 2,3,3$ & $3,4,6 / 12$ & $2 \cdot 3 \cdot 12 / 1 \cdot 4 \cdot 6$ \\
$E_{7}$ & $\mathcal{O}$ & $0 ; 2,3,4$ & $4,6,9 / 18$ & $2 \cdot 3 \cdot 18 / 1 \cdot 6 \cdot 9$ \\
$E_{8}$ & $\mathcal{I}$ & $0 ; 2,3,5$ & $6,10,15 / 30$ & $2 \cdot 3 \cdot 5 \cdot 30 / 1 \cdot 6 \cdot 10 \cdot 15$ \\
\hline
\end{tabular}

decomposition $\rho_{m}=\sum_{i=0}^{l} v_{m i} \gamma_{i}$ with $v_{m i} \in \mathbb{Z}$. We associate to $\rho_{m}$ the vector $v_{m}=\left(v_{m 0}, \ldots, v_{m l}\right)^{t} \in \mathbb{Z}^{l+1}$. As in [Kos, p. 211] we define

$$
P_{G}(t):=\sum_{m=0}^{\infty} v_{m} t^{m}
$$

This is a formal power series with coefficients in $\mathbb{Z}^{l+1}$. We also put $P_{G}(t)_{i}:=$ $\sum_{m=0}^{\infty} v_{m i} t^{m}$. Note that $v_{m 0}$ is the dimension of the $G$-invariant subspace of $S^{m}\left(\mathbb{C}^{2}\right)$. If $-I \in G$, then we have $v_{m 0}=0$ for $m$ odd. One has $-I \in G$ if and only if $\Gamma$ is not a cyclic group of odd order which is equivalent to $R=-1$. Therefore we get

$$
p_{A}\left(t^{\nu}\right)=P_{G}(t)_{0} .
$$

J. McKay [M] has observed that if $\gamma: G \rightarrow S U(2)$ is the given 2-dimensional representation of $G$ then the $(l+1) \times(l+1)$-matrix $B=\left(b_{i j}\right)$, defined by decomposing the tensor products $\gamma_{j} \otimes \gamma=\bigoplus_{i} b_{i j} \gamma_{j}$ into irreducible components, satisfies $B=2 I-C$ where $C$ is the affine Cartan matrix of the corresponding root system. Moreover, the indexing is so that the additional vertex in the extended Coxeter-Dynkin diagram corresponding to the matrix $C$ has index 0 .

Proof of Theorem 圈. From the Clebsch-Gordon formula one can derive that

$$
B v_{m}=v_{m+1}+v_{m-1}
$$

for all non-negative integers $m$ where $v_{-1}=0$ Kos, (3.3.1)]. This can be reformulated as follows (cf. [Kos, p. 222]). Let $V$ denote the set of all formal power series $x=\sum_{m=0}^{\infty} x_{m} t^{m}$ with $x_{m} \in \mathbb{Z}^{l+1}$. This is a free module of rank $l+1$ over the ring $R$ of formal power series with integer coefficients. Then $x=P_{G}(t)$ is a solution of the following linear equation in $V$ :

$$
\left(\left(1+t^{2}\right) I-t B\right) x=v_{0}
$$

Let $M(t)$ be the matrix $\left(\left(1+t^{2}\right) I-t B\right)$ and $M_{0}(t)$ be the matrix obtained by replacing the first column of $M(t)$ by $v_{0}=(1,0, \ldots, 0)^{t}$. Then Cramer's rule yields

$$
P_{G}(t)_{0}=\frac{\operatorname{det} M_{0}(t)}{\operatorname{det} M(t)}
$$

From [B, Ch. V, $\S 6$, Exercice 3] we obtain in the case when $R=-1$

$$
\operatorname{det} M(t)=\operatorname{det}\left(t^{2} I-c_{a}\right), \quad \operatorname{det} M_{0}(t)=\operatorname{det}\left(t^{2} I-c\right)
$$


where $c$ is the Coxeter element and $c_{a}$ is the affine Coxeter element of the corresponding root system. In the case $\Gamma=\mathcal{C}_{l+1}, l+1=2 n$, we assume that the numbering of $\gamma_{0}, \ldots, \gamma_{l}$ is so that the vertices of the extended Coxeter-Dynkin diagram (which is a cycle) corresponding to $\gamma_{0}, \ldots, \gamma_{n-1}$ are not connected with each other and the same holds for the vertices corresponding to $\gamma_{n}, \ldots, \gamma_{l}$. Note that this differs from the numbering in $\mathbb{B}$ but agrees with the numbering used for the discussion of the affine Coxeter element in the cases different from $A_{l}$ in [St]. (The case $A_{l}$ is excluded in that paper.) This proves Theorem 2.

For a polynomial

$$
\phi(t)=\prod_{m \mid h}\left(1-t^{m}\right)^{\chi_{m}},
$$

we use the symbolic notation

$$
\pi:=\prod_{m \mid h} m^{\chi_{m}}
$$

In the theory of finite groups, this symbol is known as a Frame shape (cf. [CN]). The Frame shapes $\pi_{A}$ corresponding to the polynomials $\phi_{A}(t)$ are indicated in Table 1.

\section{Generalizations to ICIS}

In this section we shall consider generalizations of Theorem 11 to certain ICIS.

Let $(X, x)$ be an ICIS in $\mathbb{C}^{4}$ given by quasihomogeneous equations $g=0$ and $f=0$ of degrees $d_{1}$ and $d_{2}$ respectively. As above, let $M$ be the Milnor lattice and $c: M \rightarrow M$ be the monodromy operator of $(X, x)$. By $\mathrm{GH}$ the characteristic polynomial of the monodromy operator can be computed as above using an appropriate rational function $\Phi(T)$. Similarly to the proof of Theorem 1 one can show:

Theorem 3 Let $(X, x)$ be a quasihomogeneous ICIS in $\mathbb{C}^{4}$ with weights $q_{1}, q_{2}$, $q_{3}, q_{4}$ and degrees $d_{1}, d_{2}$. Assume that $g\left(z_{1}, z_{2}, z_{3}, z_{4}\right)=z_{1} z_{4}+z_{2} z_{3}$. Define

$$
\tilde{\phi}_{A}(t):=\frac{\phi_{A}(t)\left(1-t^{d_{2}}\right)}{(1-t)^{2 g}\left(1-t^{d_{1}}\right)}, \quad \phi_{M}^{b}(t):=\frac{\phi_{M}(t)}{(1-t)} .
$$

Then we have $\tilde{\phi}_{A}^{*}(t)=\phi_{M}^{b}(t)$.

Theorem 4 Let $(X, x)$ be a quasihomogeneous ICIS in $\mathbb{C}^{4}$ with weights $q_{1}, q_{2}$, $q_{3}, q_{4}$ and degrees $d_{1}, d_{2}$. Assume that either

(A) $g\left(z_{1}, z_{2}, z_{3}, z_{4}\right)=z_{1}^{q}+z_{2} z_{3}$ and $f\left(z_{1}, z_{2}, z_{3}, z_{4}\right)=f^{\prime}\left(z_{1}, z_{2}, z_{3}\right)+z_{4}^{p}$ for some integers $p, q \geq 2$ where $q \mid d_{2}$, or

(B) $g\left(z_{1}, z_{2}, z_{3}, z_{4}\right)=z_{1}^{q}+\left(z_{2}-z_{3}\right) z_{4}$ and $f\left(z_{1}, z_{2}, z_{3}, z_{4}\right)=a z_{1}^{q}+z_{2}\left(z_{3}-z_{4}\right)$ for some $a \in \mathbb{C}, a \neq 0,1$, and some integer $q \geq 2$ and $p:=2$.

Define

$$
\begin{aligned}
\tilde{\phi}_{A}(t) & :=\frac{\phi_{A}(t)\left(1-t^{d_{2}}\right)^{p-1}\left(1-t^{\frac{d_{1}}{q}}\right)\left(1-t^{\frac{d_{2}}{p}}\right)}{(1-t)^{2 g}\left(1-t^{d_{1}}\right)\left(1-t^{\frac{d_{2}}{q}}\right)^{p}}, \\
\phi_{M}^{b}(t) & :=\frac{\phi_{M}(t)\left(1-t^{q}\right)^{p}}{(1-t)^{p-1}\left(1-t^{\langle p, q\rangle}\right)^{(p, q)}} .
\end{aligned}
$$


Then we have $\tilde{\phi}_{A}^{*}(t)=\phi_{M}^{b}(t)$.

Note that in the case $p=2, p \mid q$, the polynomial $\phi_{M}^{b}(t)$ in Theorem 4 reduces to the corresponding polynomial of Theorem 3 .

In case $(\mathrm{A})$ of Theorem $1,(X, x)$ is a $p$-fold suspension and we use the following result which can be derived from [ESt, Theorem 10]. Let $(X, 0)$ be an ICIS in $\mathbb{C}^{n+2}$ of dimension $n$ given by a map germ $F=(g, f):\left(\mathbb{C}^{n+2}, 0\right) \rightarrow$ $\left(\mathbb{C}^{2}, 0\right)$. Let $X^{\prime}=g^{-1}(0)$ and assume that $\left(X^{\prime}, 0\right)$ is an isolated singularity. Let $p \in \mathbb{N}, p \geq 2$. The $p$-fold suspension of $(X, 0)$ is the ICIS $(\tilde{X}, 0)$ defined by $\tilde{F}=(\tilde{g}, \tilde{f}):\left(\mathbb{C}^{n+2} \times \mathbb{C}, 0\right) \rightarrow\left(\mathbb{C}^{2}, 0\right)$ where $\tilde{g}(y, z)=g(y)$ and $\tilde{f}(y, z)=$ $f(y)+z^{p}$ for $(y, z) \in \mathbb{C}^{n+2} \times \mathbb{C}$. Let $\phi_{M}, \phi_{M}^{\prime}$, and $\tilde{\phi}_{M}$ be the characteristic polynomials of the monodromy operators of the singularities $(X, 0),\left(X^{\prime}, 0\right)$, and $(\tilde{X}, 0)$ respectively. Write

$$
\phi_{M}(t)=\prod_{m \mid h}\left(1-t^{m}\right)^{\chi_{m}}, \quad \phi_{M}^{\prime}(t)=\prod_{k \mid h^{\prime}}\left(1-t^{k}\right)^{\chi_{k}^{\prime}} .
$$

By ESt, loc.cit.] we have

$$
\tilde{\phi}_{M}(t)=\prod_{m \mid h} \frac{\left(1-t^{\langle m, p\rangle}\right)^{(m, p) \chi_{m}}}{\left(1-t^{m}\right)^{\chi_{m}}} \prod_{k \mid h^{\prime}}\left(1-t^{\langle k, p\rangle}\right)^{(k, p) \chi_{k}^{\prime}} .
$$

In case $(\mathrm{B}),(X, x)$ is a Brieskorn-Hamm ICIS. A Brieskorn-Hamm ICIS is a singularity $\left(V_{B}\left(w_{1}, \ldots, w_{n}\right), 0\right)$ (for integers $w_{1}, \ldots, w_{n}, w_{i} \geq 1, n \geq 3$ ) where

$$
V_{B}\left(w_{1}, \ldots, w_{n}\right):=\left\{z \in \mathbb{C}^{n} \mid b_{i 1} z_{1}^{w_{1}}+\ldots+b_{i n} z_{n}^{w_{n}}=0 ; i=1, \ldots, n-2\right\}
$$

and $B=\left(b_{i j}\right)$ is a sufficiently general $(n-2) \times n$-matrix of complex numbers. In our case $(X, x)$ is analytically isomorphic to the singularity $\left(V_{B}(q, 2,2,2), 0\right)$ for a suitable matrix $B$. The orbit invariants of a Brieskorn-Hamm ICIS are given in [NR, Theorem 2.1] and the characteristic polynomial of the monodromy operator is computed in [H].

Example 1 Let $(X, x)$ be a simply elliptic ICIS. Then $(X, x)$ is one of the singularities indicated in Table 2 (cf. [Sa1] $)$. Let $q_{1}, \ldots, q_{n}$ be the weights and $d$ be the degree of the equation(s) of $(X, x)$. Put $w_{i}=\frac{d}{q_{n+1-i}}, i=1, \ldots, n$. Then $(X, x) \cong\left(V_{B}\left(w_{1}, \ldots, w_{n}\right), 0\right)$. Hence we can apply Theorem 1 or Theorem 1 if $n=3$ or $n=4$ respectively. We obtain $\psi_{A}(t)=(1-t)^{2}, \tilde{\phi}_{A}(t)=p_{A}(t)$, and $\phi_{M}(t)=p_{A}^{*}(t)$ if $n=3$ and $\phi_{M}^{b}(t)=p_{A}^{*}(t)$ otherwise. The polynomial $\phi_{A}(t)=1+(b-2) t+t^{2}$ is the characteristic polynomial of a rotation of the Euclidian plane by the angle $\frac{2 \pi}{d}$ and hence of the Coxeter element corresponding to the Coxeter graph $\mathbb{B}$

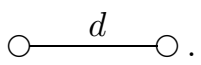

\section{References}

[B] N. Bourbaki: Groupes et algèbres de Lie, Chapitres 4,5 et 6. Hermann, Paris 1968. 
Table 2: Simply elliptic ICIS

\begin{tabular}{|c|c|c|c|c|c|}
\hline Name & $\{g ; b ;\}$ & Weights & $w_{i}$ & $\pi_{M}$ & $\pi_{A}$ \\
\hline$E_{8}$ & $\{1 ; 1 ;\}$ & $1,2,3 / 6$ & $2,3,6$ & $2 \cdot 3 \cdot 6 / 1$ & $1 \cdot 6 / 2 \cdot 3$ \\
$\tilde{E}_{7}$ & $\{1 ; 2 ;\}$ & $1,1,2 / 4$ & $2,4,4$ & $2 \cdot 4^{2} / 1$ & $4 / 2$ \\
$\tilde{E}_{6}$ & $\{1 ; 3 ;\}$ & $1,1,1 / 3$ & $3,3,3$ & $3^{3} / 1$ & $3 / 1$ \\
$\tilde{D}_{5}$ & $\{1 ; 4 ;\}$ & $1,1,1,1 / 2,2$ & $2,2,2,2$ & $2^{4} / 1$ & $2^{2} / 1^{2}$ \\
\hline
\end{tabular}

[CN] J. H. Conway, S. P. Norton: Monstrous moonshine. Bull. Lond. Math. Soc. 11, 308-339 (1979).

[D1] I. V. Dolgachev: Quotient-conical singularities on complex surfaces. Funkt. Anal. Jego Prilozh. 8:2, 75-76 (1974) (Engl. translation in Funct. Anal. Appl. 8, 160-161 (1974)).

[D2] I. V. Dolgachev: Automorphic forms and weighted homogeneous singularities. Funkt. Anal. Jego Prilozh. 9:2, 67-68 (1975) (Engl. translation in Funct. Anal. Appl. 9, 149-151 (1975)).

[D3] I. V. Dolgachev: Automorphic forms and weighted homogeneous equations. Preprint.

[D4] I. V. Dolgachev: On the link space of a Gorenstein quasihomogeneous surface singularity. Math. Ann. 265, 529-540 (1983).

[D5] I. V. Dolgachev: Mirror symmetry for lattice polarized K3 surfaces. J. Math. Sci. 81, 2599-2630 (1996).

[ESt] W. Ebeling, J. H. M. Steenbrink: Spectral pairs for isolated complete intersection singularities. J. Alg. Geom. 7, 55-76 (1998).

[GDC] S. M. Gusein-Zade, F. Delgado, A. Campillo: On the monodromy of a plane curve singularity and the Poincaré series of the ring of functions on the curve. Funkt. Anal. Jego Prilozh. 33:1, 66-68 (1999) (Engl. translation in Funct. Anal. Appl. 33:1, 56-57 (1999)).

[GH] G.-M. Greuel, H. Hamm: Invarianten quasihomogener vollständiger Durchschnitte. Invent. math. 49, 67-86 (1978).

[H] H. Hamm: Exotische Sphären als Umgebungsränder in speziellen komplexen Räumen. Math. Ann. 197, 44-56 (1972).

[Kn] H. Knörrer: Group representations and the resolution of rational double points. In: Finite groups - Coming of Age, Proceedings, Montreal 1982 (J. McKay, ed.), Contemporary Mathematics, Vol. 45, Am. Math. Soc., Providence 1985, pp. 175-222.

[Kos] B. Kostant: The McKay, correspondence, the Coxeter element and representation theory. In: Élie Cartan et les mathématiques d'aujourd'hui (Lyon, 1984), Astérisque, Numéro hors série, 1985, pp. 209-255.

[M] J. McKay: Graphs, singularities, and finite groups. Proc. Symp. Pure Math. Vol. 37, 183-186 (1980). 
[MO] J. Milnor, P. Orlik: Isolated singularities defined by weighted homogeneous polynomials. Topology 9, 385-393 (1970).

[NR] W. D. Neumann, F. Raymond: Seifert manifolds, plumbing, $\mu$-invariant and orientation reversing maps. In: Algebraic and Geometric Topology, Proceedings, Santa Barbara 1977 (K. C. Millet, ed.), Lect. Notes in Math., Vol. 664, Springer, Berlin, Heidelberg, New York, 1978, pp. 162196.

[OW] P. Orlik, P. Wagreich: Algebraic surfaces with $k^{*}$-action. Acta math. 138, 43-81 (1977).

[P] H. Pinkham: Normal surface singularities with $\mathbb{C}^{*}$ action. Math. Ann. 227, 183-193 (1977).

[Sa1] K. Saito: Einfach-elliptische Singularitäten. Invent. math. 23, 289-325 (1974).

[Sa2] K. Saito: Regular system of weights and associated singularities. In: Complex Analytic Singularities (T. Suwa, P. Wagreich, eds.), Adv. Studies in Pure Math 8, North-Holland, Amsterdam etc., 1987, pp. 479526.

[Sa3] K. Saito: On a duality of characteristic polynomials for regular systems of weights. Preprint RIMS, Kyoto University 1994.

[Sa4] K. Saito: Duality for regular systems of weights: a précis. In: Topological Field Theory, Primitive Forms and Related Topics (M. Kashiwara, A. Matsuo, K. Saito, I. Satake, eds.), Progress in Math., Vol. 160, Birkhäuser, Boston Basel Berlin, 1998, pp. 379-426.

[Sp] T. A. Springer: Poincaré series of binary polyhedral groups and McKay's correspondence. Math. Ann. 278, 99-116 (1987).

[St] R. Steinberg; Finite subgroups of $S U_{2}$, Dynkin diagrams and affine Coxeter elements. Pac. J. Math. 118, 587-598 (1985).

[W1] P. Wagreich: Algebras of automorphic forms with few generators. Trans. AMS 262, 367-389 (1980).

[W2] P. Wagreich: Automorphic forms and singularities with $\mathbb{C}^{*}$-action. Illinois J. Math. 25, 359-382 (1981).

[W3] P. Wagreich: The structure of quasihomogeneous singularities. Proc. Symp. Pure Math Vol. 40, Part 2, 593-611 (1983). 